\title{
Gestante Bariátrica
}

- As vantagens da cirurgia bariátrica para as mulheres obesas que desejam engravidar incluem aumento da taxa de fertilidade; redução das complicações metabólicas como diabetes gestacional e síndromes hipertensivas da gestação; e redução de algumas complicações fetais, como macrossomia fetal;

- Apesar do aumento na taxa de fertilidade, as gestantes previamente submetidas à cirurgia bariátrica são consideradas um grupo de alto risco e as complicações da cirurgia bariátrica podem se desenvolver ou se exacerbar durante a gestação;

- De forma geral, a gestação entre mulheres que já se submeteram à cirurgia bariátrica é segura. Ocorre a redução no risco de diabetes gestacional e síndromes hipertensivas como a pré-eclampsia, reduz o risco de crianças nascidas grandes para a idade gestacional, porém a cirurgia aumenta o risco de prematuridade e neonatos pequenos para a idade gestacional (PIG);

- Algumas deficiências nutricionais podem impactar negativamente a saúde materna e fetal podendo, inclusive, favorecer a ocorrência de má formações congênitas, como a deficiência de vitaminas B9, A, K, D, B12, além de zinco e cálcio;

- O impacto nutricional da cirurgia bariátrica irá depender da técnica cirúrgica empregada. Nas técnicas restritivas e disabsortivas, como o bypass gástrico em Y de Roux, ocorre maior comprometimento digestivo e absortivo quando comparado às técnicas puramente restritivas como a gastrectomia vertical ou sleeve;

- Um estudo brasileiro demonstrou que em todos os trimestres gestacionais, mais de $60 \%$ das 30 mulheres previamente submetidas à cirurgia bariátrica apresentaram níveis inadequados de retinol ou beta-caroteno, seja uma deficiência leve ou moderada. Além disso, a cegueira noturna esteve presente em 57\% dessa amostra;

- Um estudo brasileiro demonstrou que em uma população de 46 gestantes previamente submetidas à cirurgia bariátrica por bypass em Y Roux, a prevalência de inadequação de vitamina D foi superior a 70\% atingindo $82 \%$ no $3^{\circ}$ trimestre gestacional. A suplementação com 800 UI de colecalciferol ao dia durante toda a gestação não foi suficiente;

- As síndromes de dumping são comuns após a cirurgia bariátrica e podem apresentar desafios diagnósticos e terapêuticos na gravidez. As medidas dietéticas são o pilar na terapêutica das síndromes de dumping;

- Considerações importantes no atendimento da gestante bariátrica: Qual o tipo de cirurgia realizada? Quando foi o procedimento cirúrgico? Qual a perda de peso obtida? Avaliação das deficiências nutricionais; uso de suplementos vitamínicos/minerais; uso de probióticos; e cuidado com o risco de hipoglicemia, hiperinsulinemia e dumping.

\section{Referências bibliográficas}

1. MAGGARD, M.A. et al. Pregnancy and fertility following bariatric surgery: a systematic review. JAMA; 300(19):2286-96, 2008.

2. HARRIS, A.A.; BARGER, M.K. Specialized care for women pregnant after bariatric surgery. J Midwifery Womens Health; 55(6):529-39, 2010.

3. CARREAU, A.M.; NADEAU, M.; MARCEAU, S. et al. Pregnancy after Bariatric Surgery: Balancing Risks and Benefits. Can J Diabetes; 41(4):432438, 2017.

4. JANS, G.; MATTHYS, C.; BOGAERTS, A. et al. Maternal micronutrient deficiencies and related adverse neonatal outcomes after bariatric surgery: a systematic review. Adv Nutr; 6(4):420-9, 2015.

5. GILBERT, E.W.; WOLFE, B.M. Bariatric surgery for the management of obesity: state of the field.

Plast Reconstr Surg; 130:948-954, 2012.

6. CHAGAS, C.; SAUNDERS, C.; PEREIRA, S. et al. Vitamin A status and its relationship with serum zinc concentrations among pregnant women who have previously undergone Roux-en-Y gastric bypass. Int J Gynaecol Obstet; 133(1):94-7, 2016.

7. MEDEIROS, M.; MATOS, A.C.; PEREIRA, S.E. et al. Vitamin D and its relation with ionic calcium, parathyroid hormone, maternal and neonatal characteristics in pregnancy after roux-en-Y gastric bypass. Arch Gynecol Obstet; 293(3):539-47, 2016.

8. KASSIR, R.; GOISET, M.P.; TIFFET, O. Pregnancy Following Bariatric Surgery-Medical Complications and Management. Obes Surg; 26(11):2785, 2016. 